\title{
Two-Step Scheduling Scheme to Support Fairness and QoS in DVB-S2 System
}

\author{
ManKyu Park ${ }^{1}$, DongBae Kang ${ }^{2}$, and DeockGil Oh ${ }^{1}$ \\ ${ }^{1}$ Aerospace ICT research Department, Electronics and Telecommunications Research Institute \\ 218 Gajeongno, Yuseong-gu, Daejeon 305-700, KOREA \\ [e-mail: \{neomkpark, dgoh $\} @$ etri.re.kr] \\ ${ }^{2}$ Agency for Defense Development \\ Bugyuseong daero 488 beon gil, Yuseong, Daejeon 305-152, KOREA \\ [e-mail: skylove7890@gmail.com] \\ *Corresponding author: ManKyu Park
}

Received April 17, 2014; revised June 12, 2014; accepted October 22, 2015;

published November 19, 2015

\begin{abstract}
The use of an efficient packet scheduling scheme for a forward link in satellite communication networks is very important to support fairness for each return channel satellite terminal (RCST) and the service differentiations for user traffics. To support fairness and QoS for each RCST with service-level agreement (SLA), the Adaptive Coding and Modulation (ACM) system in a satellite hub has to process packets with considering modulation and coding (MODCOD) and packet types. Although a DVB-S2 system with ACM scheme has higher transmission efficiency, it cannot offer fairness or quality of service (QoS) to RCSTs. Because the data are transmitted with high MODCOD in regions with clear skies, while data are transmitted using low MODCOD in regions experiencing rain events. In this paper, we propose a two-step scheduling scheme offering fairness and QoS to RCSTs, while minimizing a decrease in throughput. The proposed scheme is carried out performance evaluations using a computer simulation. As results of this simulation, the proposed scheduler was shown to support bandwidth fairness to an individual RCST, and provide a level of QoS differentiation for user traffics.
\end{abstract}

Keywords: DVB-S2, ACM, Fairness, Scheduling, QoS

A preliminary version of this paper appeared in IEEE ICCE 2013, Jan 11-14, Las Vegas, , NV, USA. This version includes a concrete mathematical analysis and valueable simulation results for the proposed scheme. This research was funded by the MSIP(Ministry of Science, ICT \& Future Planning), Korea in the ICT R\&D Program 2015 


\section{Introduction}

The DVB-S2 standard [1]-[3] including ACM scheme was approved in 2004 to support satellite broadcasting, as well as interactive and professional applications. This makes it possible to achieve a low-cost, near Shannon bound forward error correction (FEC) performance combined with a wide range of modulation and coding schemes [4] and it can provide an increase in transmission performance of up to $30 \%$ compared to the previous DVB-S [5] modem standard using the same bandwidth and power under the same channel conditions. Satellite communication system supporting ACM has a capacity that varies over time as available resources dynamically adapt to the channel and system conditions, thus dramatically increasing the overall satellite network capacity.

However ACM scheme has higher bandwidth efficiency, it cannot offer fairness to each RCST on the ground. Because the data are transmitted with high MODCOD in regions under clear skies, and the data are transmitted with low MODCOD in regions experiencing rain events. In fact, this situation is very irrational for RCSTs which have the same SLA contract. Therefore ACM scheme in DVB-S2 needs to control the packet processing using fairness mechanism.

ACM can change the transmission capability of the modem at the physical layer to enhance the link availability. When ACM selects a low MODCOD for a poor channel condition, the current transmission capability will be reduced and packets will be buffered in the IP or MAC queue, and finally some packets will be dropped in the drop-tail queue of the IP or link layer. Under this situation, if there is no QoS control mechanism for dropped packets, important packets may be discarded. To avoid the loss of important packets, a scheduler in the IP layer should support QoS [6][7].

In this paper, we propose a two-step scheduling scheme to offer fairness and QoS for RCSTs, while minimizing the decrease in throughput. In our proposed scheme, QoS control is adopted at the IP layer to support service differentiations for user traffics, and a fairness support function is provided at the link layer to share the available channel resource in a fair manner. The proposed scheme is carried out performance evaluations using a computer simulation. As results of this simulation, the proposed scheme is shown that bandwidth fairness is supported for the individual RCST and provides some level of QoS differentiation between the best efforts (BE), assured forward (AF), and expedited forward (EF) classes [8].

The remainder of this paper is organized as follows. In section 2, ACM scheme is described in DVB-S2 and briefly introduce our reference service model. The architecture of the proposed scheme is presented in section 3 , which also describes the operation conducted at each step. We describe the results of the simulation and a performance evaluation in section 4 . In section 5, the main conclusions of this proposal and further research topics are outlined.

\section{Related Works}

\subsection{DVB-S2 ACM scheme overview}

The DVB-S2 modulator operates at a constant symbol rate, since the downlink carrier bandwidth is assumed to be constant. A sequence of physical layer frames multiplexed in a TDM fashion is transmitted. Each frame transports a coded block and adopts a uniform modulation format. However, when ACM is implemented, the coding scheme and modulation 
format may change frame by frame. Fig. 1 shows the ACM operation with DVB-S2/RCS2. The architecture of an satellite networks are commonly composed by the network control center (NCC), with RCST connected to the NCC through a return link. In the ACM system, satellite terminals allocated under clear sky conditions are characterized as having high link bandwidth availability. Therefore, these terminals will be provided with a high-level MODCOD. In contrast, terminals allocated under a heavy rain are characterized as having reduced link bandwidth availability. And these terminals will be provided with a low-level MODCOD, which includes a robust channel protection mechanism and a specific modulation scheme. As a result, the bandwidth capacity will fluctuate between the minimum and maximum values, since the ACM mechanism will work continuously to mitigate atmospheric impairments [9].

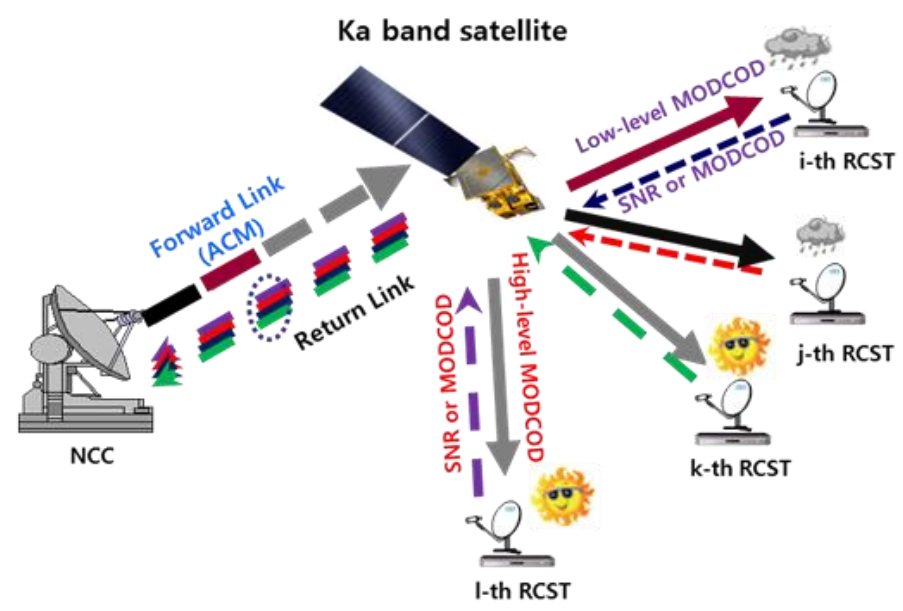

Fig. 1. ACM operation over DVB-S2/RCS2 network

The ACM scheme in DVB-S2 is used for enhancing the connectivity maximization to use variable information bits of payload according to a certain MODCOD. Fig. 2 shows how ACM works according to the channel conditions. If the wireless link is in a good condition under a clear sky, a large amount of data can be transmitted with high MODCOD. On the other hand, if the wireless link is experiencing poor conditions with heavy rain, the amount of transmitted data are not high compared to under a clear sky, because redundant data were also included for robustness.

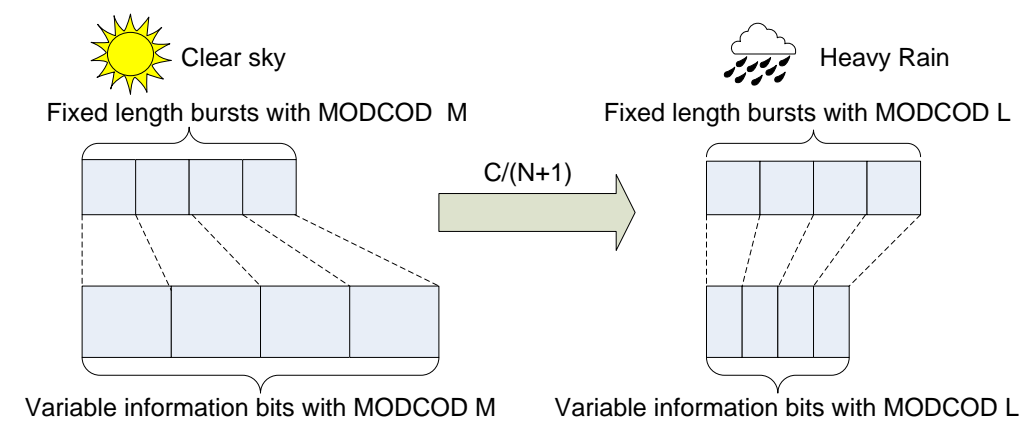

Fig. 2. ACM scheme according to MODCOD 


\subsection{ACM effect on TCP performance}

ACM is a standard technique for DVB-S2, it is used to best match the end to end connection according to its channel condition and this is considered to be best solution to overcome link impairment such as rain attenuation. However, if modem transmission capability is changed by ACM operation, congestion control problem is experienced between a modem and transport layer protocol, especially when TCP is used for transport layer protocol.

Fig. 3 is shown for the TCP congestion control problem, a modem with ACM operates dynamically according to channel condition. If the wireless link is in good condition with clear sky, a modem selects a high MODCOD then TCP's sending rate is increased with a suitable Congestion window (cwnd). However if the wireless link is in bad condition with heavy rain, a modem is adopted a low MODCOD to provide robustness for packets.

Although TCP can be actively responded to changes of network capability using its congestion control mechanisms, significant changes of bandwidth delay product (BDP) caused by ACM can still bring severe problems to networks which are involved with buffer in systems, because the TCP in transport layer does not recognize such a sudden change of the access network in lower layer. In this situation, the most significant problem is packet loss from buffer overflow. Buffer overflow can be experienced when bandwidth is changed from a high bandwidth with a high MODCOD to a low bandwidth with a low MODCOD. At this moment TCP does not recognize the bandwidth change without information from IP, MAC or PHY layer. This is reason why QoS control needs for ACM system [10][11].

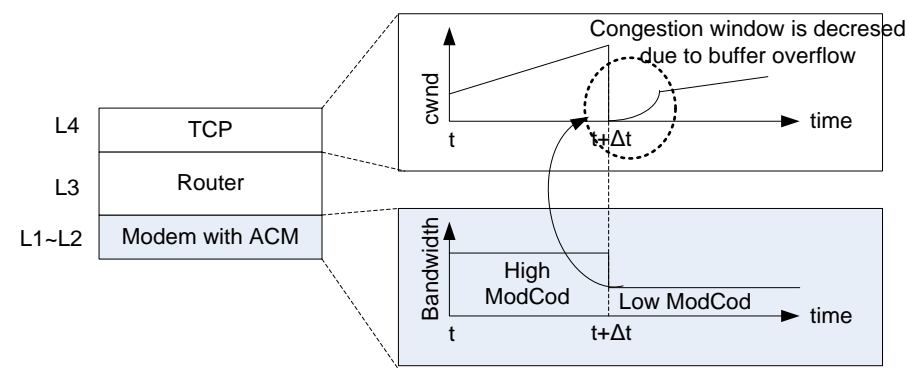

Fig. 3. TCP congestion control problem due to ACM operation

\subsection{BSM QoS mechanism based on DiffServ}

The broadband satellite multimedia (BSM) working group has defined a QoS functional architecture, ETSI-BSM-QoS, standardized [12] to provide a variety of services over satellite networks with QoS. This framework establishes the required QoS mechanisms to define the priorities among users and applications based on the DiffServ architecture [13]. The QoS mechanism supporting DiffServ allows the EF traffic class to have the highest priority, while the $\mathrm{AF}$ traffic class has higher priority than the $\mathrm{BE}$ traffic class. As a result, the $\mathrm{BE}$ traffic class, which is based on a best effort scheme, uses the remaining link capacity, as it is able to use any bandwidth unused by other classes.

As shown in Fig. 4, at a BSM satellite terminal, the QoS control functions operate in the control plane protocol stack across the satellite independent-service point (SI-SAP), and interact with the user plane. The IP resource manager handles IP queues and IP resource requests, and passes them to the lower layers if necessary; it also interfaces with the external IP 
signaling protocols such as Resource reservation protocol (RSVP). Queue identifiers (QIDs) are considered to be controlled locally by the QID resource manager, which directly interfaces with the satellite dependent (SD) module managing real satellite resources [12].

Our proposed architecture is fully compliant with the ETSI BSM SI-SAP interface because the proposed architecture keeps SD module and SI architectures separate. To support this architecture, Differentiated service code points (DSCPs) in the IP layer QoS need to be mapped to the satellite-dependent class of service, which can be archived for the use of an ETSI BSM SI-SAP interface. In addition, the proposed architecture is applied the priority scheduling algorithm for the IP layer in the first step scheduler. The proposed architecture of two-step scheduler supports the DiffServ model to provide the QoS to each RCST in a satellite networks, multiplexing a variety of packets. The proposed scheduler is described minutely in section 3.

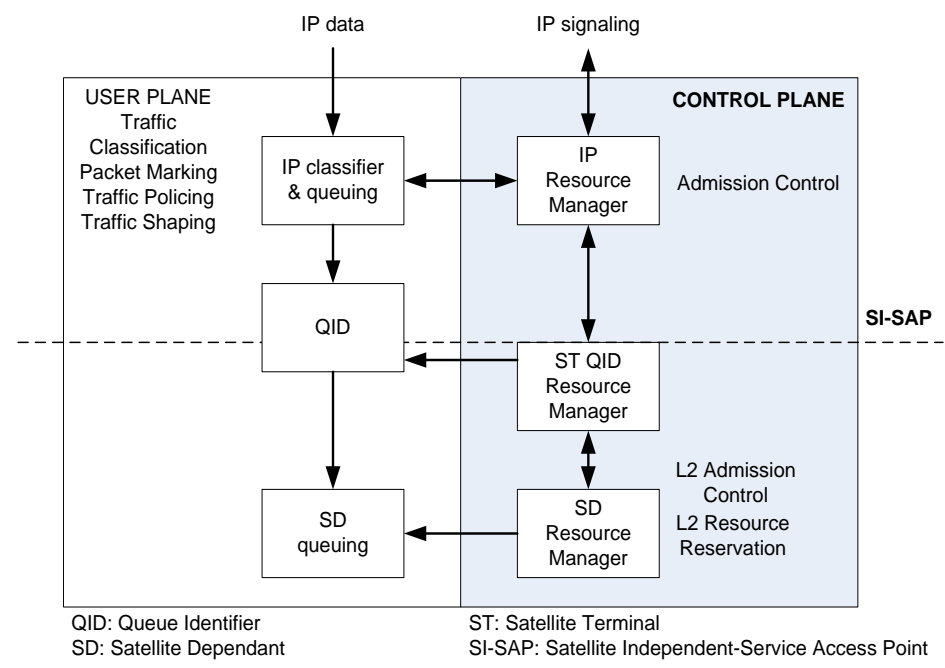

Fig. 4. ETSI BSM functional architecture [12]

\subsection{Scheduling algorithms for Fairness}

What is more important factor between fairness and system efficiency? From a network operator perspective, it is very important to use the channel efficiently and the high system efficiency because the radio resources are limited and the revenue should be maximized. On the other hand, from the users' point of view, it is more important to have fair resource allocation such that they are not in a starvation/outage situation and their QoS requirements are guaranteed [14]. Thus, it is difficult to decide what factor is more improtant between the system efficiency and user's faireness.

Because of this controversy, [15] proposed propotional fairness scheme as the practical soultion. In this study, propotional fairness achieves a good tradeoff between efficiency arnd fairness.

Recently, a variety of optimization theory [16][17] such as game theory, utility function, cost function, convex theory, etc. applied to provide fairness. Also [6] proposed a cross-layer queuing architecture and an adaptive scheduling policy that supports different fairness policies.

However, as we previously described that if users have the same SLA and pay the same cost, users should have the same service quality. To meet the goal, in this paper, we just focus 
on fairness and supporting QoS according to traffic types. Table $\mathbf{1}$ is shown various fair queuing algorithms, we can use these algorithms to offer the same opportunity to use common resources for users.

Table 1. Performance of Fair Queuing Algorithms [18]

\begin{tabular}{|c|c|c|}
\hline Algorithm & Fairness Measure & Work Complexity \\
\hline Round Robin & $\infty$ & $\mathrm{O}(1)$ expected \\
\hline Fair Queuing & Max & $\mathrm{O}(\log (\mathrm{n}))$ \\
\hline Self-clocked Fair Queuing & 2 Max & $\mathrm{O}(\log (\mathrm{n}))$ \\
\hline Deficit Round Robin & 3 Max & $\mathrm{O}(1)$ expected \\
\hline
\end{tabular}

\section{Proposed Scheme}

\subsection{Reference service model}

DVB-S2 satellite networks are mostly used for either broadcasting or Internet access services. In this study, target customer service is Internet access services only, as well as our proposed reference model for supporting a level of QoS differentiation in a satellite communication system, as shown in Fig. $\mathbf{5}$.

In our reference model, DVB-S2 is used for the forward link, and DVB-RCS2 is used for the return link. Networks are composed of three sources which send traffic data to a remote destination node. Each source node supports different services having predefined per-hop behaviors (PHBs). The EF class is represented by a real-time voice over IP (VoIP) service employing the user data protocol (UDP). The AF class represents Web services employing the transmission control protocol (TCP). The BE class represents a bulk data transfer generated by a persistent file transfer protocol (FTP) service using TCP.

To support QoS in this model for a variety of traffic types and various link bandwidths with ACM, The forward link for satellite networks should support fairness among RCSTs, and it also has to provide the QoS based on DiffServ according to the traffic characteristics in a wired LAN area.

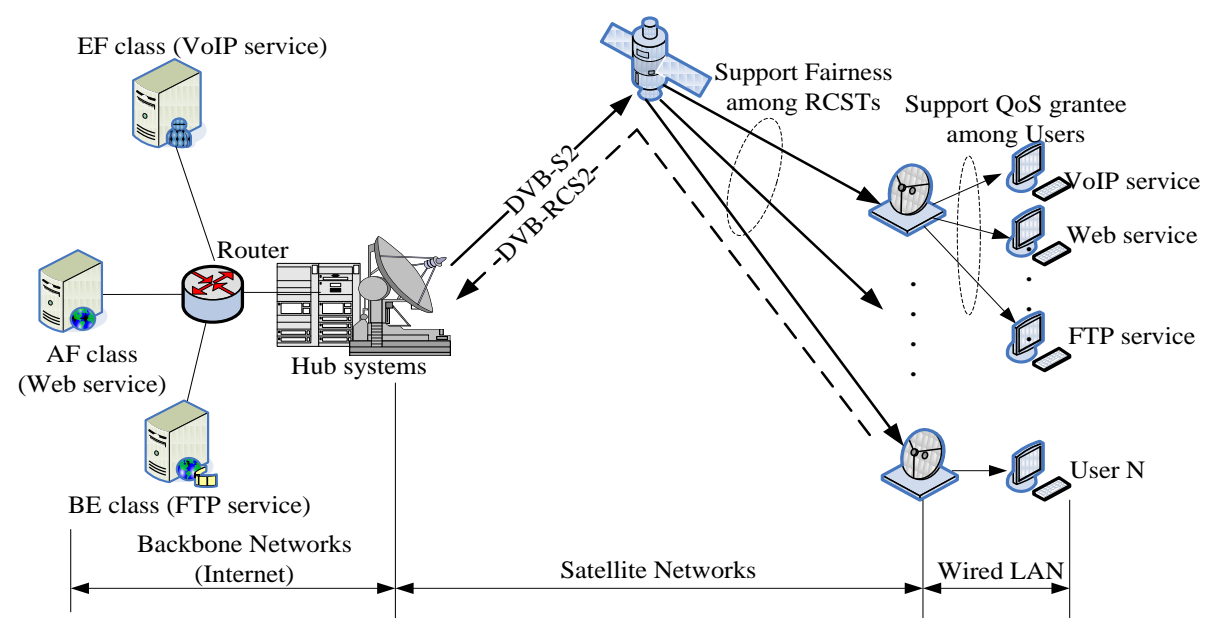

Fig. 5. Proposed reference model for QoS and fairness 


\subsection{Proposed system and scheduler architecture}

In this section, we present the architecture and scheduler for proposed scheme. To use ACM scheme, an ACM system should be added in the NCC. Fig. 6 indicates the composition of the ACM system. The ACM system consists of an ACM routing section and a control section. The ACM routing section receives IP data traffic of the forward link from the router, and forward link signaling (FLS) packets from the dynamic resource management module (DRM).

The ACM router in the ACM routing section consists of blocks of a packet classifier, input buffers, ACM signaling inserts, and a scheduler, as shown in Fig. 6. The user data packets received through the input interface are transmitted to the packet classifier block. In the packet classifier block, packets are classified based on the DiffServ QoS mechanism, and are transmitted to the input buffers for providing the QoS. After transferred packets are stored in the input buffers, the IP data are converted into GSE, and ACM signaling is inserted. The data are then classified according to the MODCOD level, and converted into traffic data formats for ACM transmission. The ACM control section receives the signal-to-noise plus interference ratio (SNIR) information at each RCST, and transmits the MODCOD level determined by the received SNIR information.

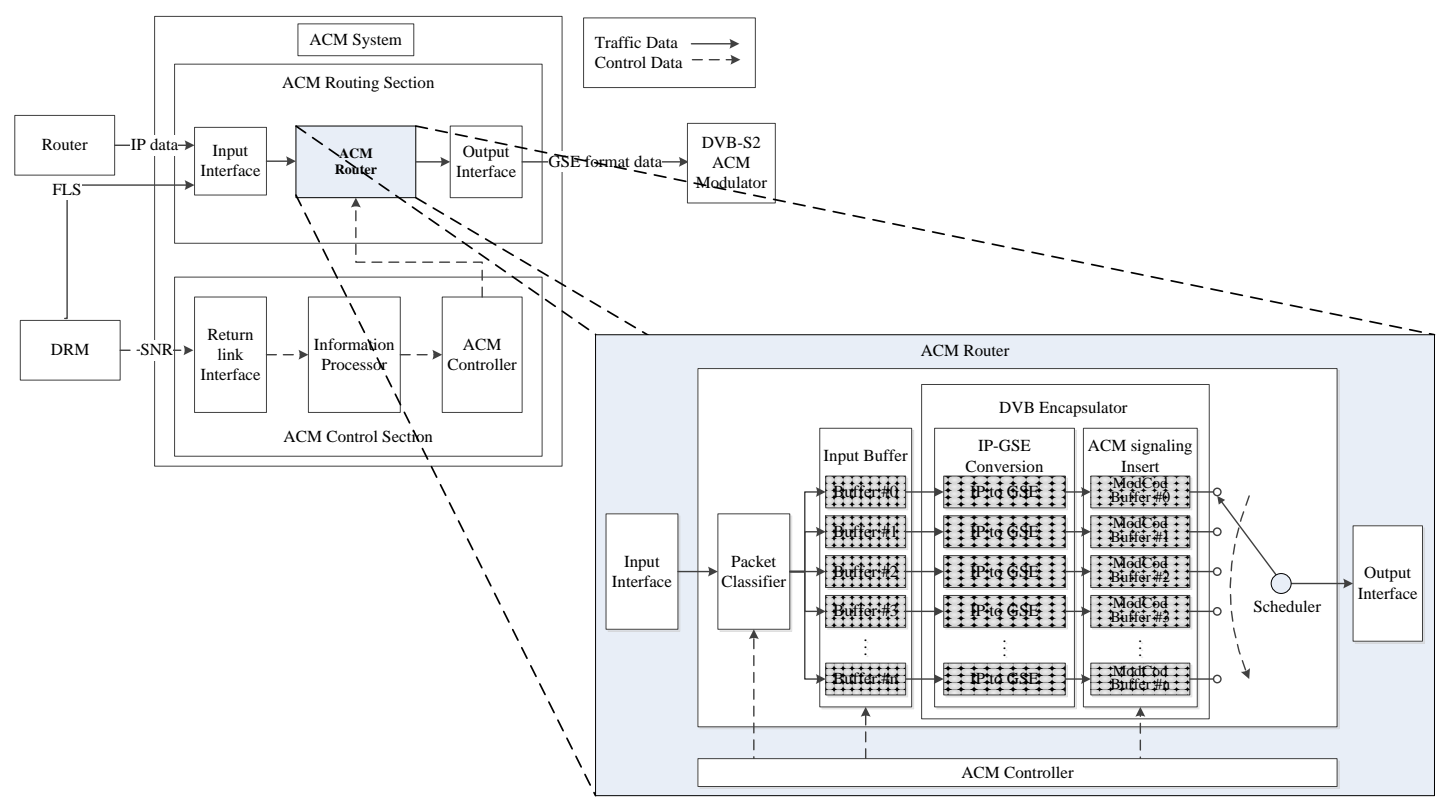

Fig. 6. Proposed ACM system structure

Scheduler architecture for supporting fairness and QoS is indicated in Fig. 7. The packets are classified as being either of the EF class, AF class, or BE class of QoS in the packet classifier. Classified packets are processed by the IP layer QoS scheduler, and transferred to the MODCOD queues. To support fairness to earth stations, MODCOD scheduler processes the packets by providing low MODCOD queues with more timeslots. MODCOD queues are controlled by the link layer fairness scheduler. 


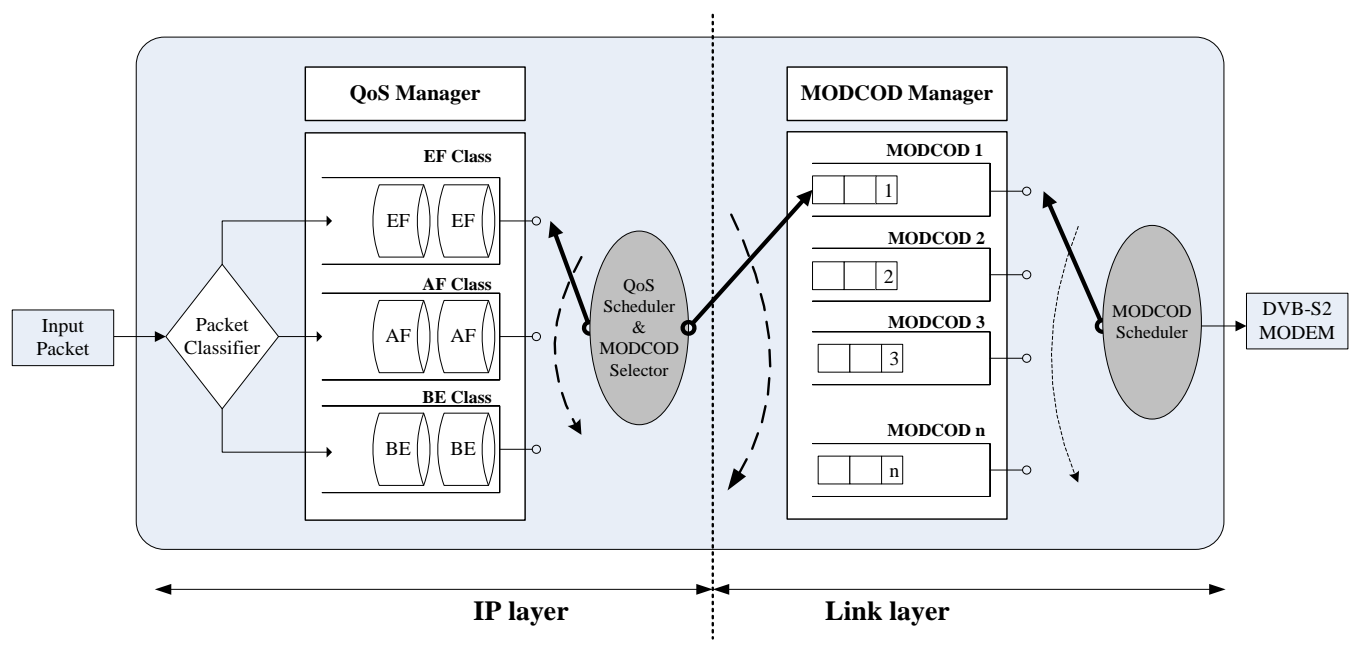

Fig. 7. Proposed scheduler architecture at IP and link layer

Priority scheduling is used to support QoS at IP-layer, its scheduler can be used to provide service differentiation according to the user traffic types. When satellite communication systems adopt an ACM scheme the transmission capability is changed dynamically according to the link conditions. It means that data throughputs are various, and sometimes packets will be lost owing to a lack of bandwidth. The proposed IP-layer QoS scheduler, which supports priority scheduling, can select the packets to drop based on the traffic preference and QoS policy.

This scheduling algorthm is easliy analyzed the performance using the queuing theory. To solve the M/D/1 queuing system, we start the Pollaczek-Khinchin (P-K) formula to derive the result of the $\mathrm{M} / \mathrm{G} / 1$ formula. Because $\mathrm{M} / \mathrm{D} / 1$ is a special case of $\mathrm{M} / \mathrm{G} / 1$, we can achieve an $\mathrm{M} / \mathrm{D} / 1$ solution using the $\mathrm{M} / \mathrm{G} / 1$ case in [19].

The Pollaczek-Khinchin (P-K) formula is given through the following equation.

$$
\text { Average service time }=\bar{X}=E[X]=1 / \mu
$$

In addition, variance $\sigma^{2}$ and arrivals follow a Poisson process with parameter $\lambda$. The second moment of service time is also given by

$$
\overline{X^{2}}=E\left[X^{2}\right]=\sigma^{2}+\frac{1}{\mu^{2}}
$$

The average waiting time using the $\mathrm{P}-\mathrm{K}$ formula is then

$$
E(W)=\frac{\lambda \overline{X^{2}}}{2(1-\rho)}
$$

where $E(W)$ is the expected customer waiting time in a queue in the $M / G / 1$ queuing system, and $\rho=\lambda / \mu=\lambda \bar{X}$ and is used for system utilization.

Based on Little's theorem, the average number of packets in the queue is given by 


$$
E(q)=\frac{\lambda^{2} \overline{X^{2}}}{2(1-\rho)}
$$

The system needs priority queuing modeling for the IP-layer QoS scheduler, and we therefore apply the queuing theory of non-preemptive priority queues in the $M / G / 1$ system using [20].

Let us assume that we have $n$ classes, each with Poisson arrivals of parameters $\lambda_{1}, \cdots$, $\lambda_{n}$, and general service time distributions with an average of $1 / \mu_{1}, \cdots, 1 / \mu_{n}$, and variances of $\sigma_{1}{ }^{2}, \cdots, \sigma_{n}{ }^{2}$. The average waiting time for class $i, i=1, \cdots, n$, is given by

$$
\left(W_{i}\right)=\frac{E\left(T_{0}\right)}{\left(1-\sum_{j=1}^{i} \rho_{j}\right)\left(1-\sum_{j=1}^{i-1} \rho_{j}\right)}
$$

with $\rho_{j}=\lambda_{j} / \mu_{j}$, and

$$
E\left(T_{0}\right)=\frac{\lambda E\left[X^{2}\right]}{2}=\sum_{j=1}^{n} \lambda_{j} \frac{E\left[X_{j}^{2}\right]}{2}=\sum_{j=1}^{n} \lambda_{j} \frac{\overline{X_{J}^{2}}}{2}
$$

with $\rho=\sum_{j=1}^{n} \rho_{j}, \lambda=\sum_{j=1}^{n} \lambda_{j}$, and $\overline{X_{J}^{2}}=\sigma_{j}^{2}+\left(1 / \mu_{j}\right)^{2}$

$$
E\left(q_{i}\right)=\lambda_{i} E\left(W_{i}\right) \frac{\lambda_{i} E\left(T_{0}\right)}{\left(1-\sum_{j=1}^{i} \rho_{j}\right)\left(1-\sum_{j=1}^{i-1} \rho_{j}\right)}
$$

We apply the priority scheduling algorithm to deal with packets that have other QoS levels. In the priority scheduler such as Fig. 8, packets from a high-priority queue are always processed before packets from a medium-priority queue. Likewise, packets from a medium-priority queue are always processed before packets from a low-priority queue.

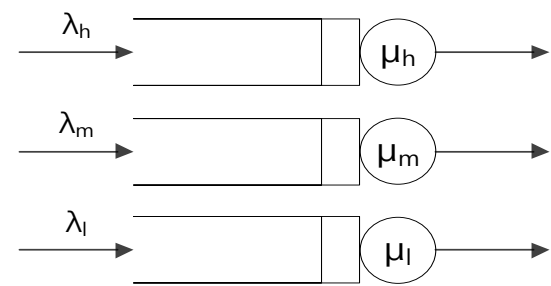

Fig. 8. Prority scheduler for QoS

We proposed the fairness improvement-weighted round robin (FI-WRR) scheduler to provide the throughput fairness, the proposed scheme focuses solely on fairness among the RCSTs. There are many kinds of criteria used to calculate the weighting factor to provide fairness, such as the mean packet counts and queue size. In this paper, we consider the maximum capacity of the DVB-S2 forward link and the available capacity of the current MODCODs. The packets processed by the second step scheduler are classified by each MODCOD. Each MODCOD queue is processed using a weighted round-robin scheduling 
algorithm to provide fairness for the RCSTs. The required timeslots are calculated according to the following steps:

The total time slot counts, $t s_{t o t}$, for a system are determined with a symbol rate at a physical layer considering a certain frequency band.

The fairly allocated timeslots for each RCST, $n_{r}^{t s}$, are

$$
n_{r}^{t s}=\frac{t s_{t o t}}{N_{\text {logged_RCST }}}
$$

The available bandwidth, $B W_{a v}$, is

$$
B W_{a v}=c_{0} \cdot n_{r}^{t s}+c_{1} \cdot n_{r}^{t s} \cdots+c_{n-1} \cdot n_{r}^{t s}=\sum_{i=0}^{n-1} c_{i} \cdot n_{r}^{t s}
$$

where $c_{i}$ is the transmission capability of the modem according to a certain MODCOD.

The available bandwidth for each RCST, $\overline{B W_{a v}}$, is

$$
\overline{B W_{a v}}=\frac{B W_{a v}}{N_{r c s t}}
$$

The required bandwidth of the $i$-th RCST for fair resource allocation is

$$
r e q_{-} b w_{i}=\overline{B W_{a v}}-c_{i} \cdot n_{r}^{t s}
$$

The required timeslots for the $i$-th RCST is calculated as

$$
n_{i}^{t s}=\left\lfloor\frac{r e q_{-} b w_{i}}{c_{i}}\right\rfloor
$$

RCSTs in regions experiencing bad weather conditions are allocated more timeslots than RCSTs in regions under clear sky conditions. Users located in a region with clear sky conditions share a time slot allocated to themselves with users located in a region with bad weather conditions. While users located in a region with bad weather conditions transmit packets at a low transmission rate, they have more time slots than users located in a region with clear sky conditions. Therefore, although they have different transmission rates in a separate location, they offer RCSTs having fairness with respect to different weather conditions.

By applying this algorithm, we can offer the same throughput to all RCSTs. However, the overall throughput decreases because users belonging to a region with clear sky conditions will be affected by poor weather conditions. For a bad link caused by poor weather condition, the forward link experiences a capacity reduction, which means that the total transmitted packet counts are decreased. However, in this paper, we apply the priority scheduling algorithm in the first step scheduler to minimize the decrease in the total throughput efficiency with fairness. By 
applying the priority scheduling algorithm in the first step scheduler, BE class packets was lost firstly and important packets, $\mathrm{EF}$ and $\mathrm{AF}$ class, are transmitted safely.

\section{Performance Evaluations}

The proposed scheme is verified through computer simulations using sim++ [21] which is a queuing simulator. The simulation configurations are shown in Table 2. The queuing network topology used for the simulation is shown in Fig. 9. Here, three sources send data to a remote destination. Each source node supports different services, having a predefined PHB[22]. We apply the first-step algorithm with pure round-robin and priority queuing algorithms to ensure QoS support.

Table 2. Simulation Configurations

\begin{tabular}{|c|c|}
\hline Simulation Parameters & Values \\
\hline \hline Simulation time & 100sec \\
\hline Bandwidth & 120Mbps $\sim$ 300Mbps \\
\hline $\begin{array}{c}\text { Propagation delay } \\
\text { for DVB-S2 link }\end{array}$ & $\begin{array}{c}\text { 5ms (wired link for Hub system) } \\
250 \text { ms (satellite link) } \\
10 \mathrm{~ms} \text { (wired link for VSAT) }\end{array}$ \\
\hline Traffic packet size & 1500 bytes \\
\hline Input traffic ratio & $1: 1: 2$ (EF:AF:BE) \\
\hline & MODCOD $8(\mathrm{QPSK}, 4 / 5)$ \\
MODCODs & MODCOD 13 (8PSK, 2/3) \\
for ACM & MODCOD 19 (16APSK, 3/4) \\
& MODCOD 25 (32APSK, 4/5) \\
\hline
\end{tabular}

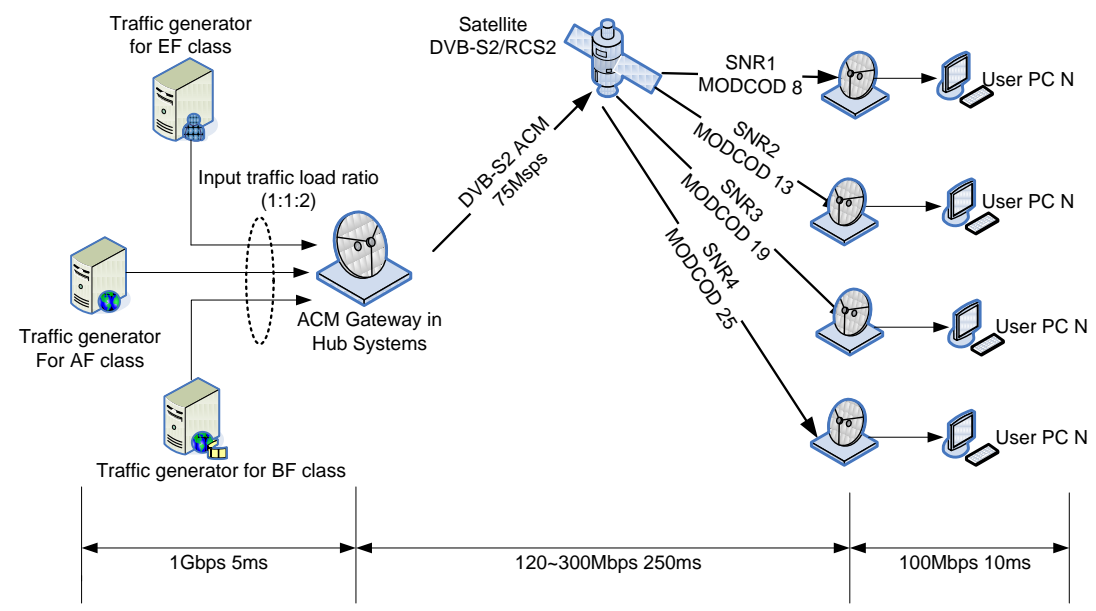

Fig. 9. Network topology

\subsection{Average packet delay}

We analyze the average packet delay using the round-robin scheduling algorithm, as shown in Fig. 10. The average packet delay of the round-robin scheduling algorithm is similarly 
retained regardless of the QoS classes, because the scheduler based on round-robin algorithm has the same weight value and processes the same amount of packets during the same time period. Looking at Fig. 10 in detail, BE packets are still slightly longer delay than other packets but that delay time is very short and negligible. Because a lot of BE packets, of course some $\mathrm{AF}$ and $\mathrm{EF}$ packets are also included, are discarded in the drop-tail queue.

Therefore, a packet scheduling algorithm is needed to provide the priority such as the weighted round-robin or priority scheduling algorithm. To support QoS in IP layer, we apply the priority scheduling algorithm in the first scheduler. As shown in Fig. 11, the average packets delay of EF and AF class traffics which has high priority are significantly decreased because the packets are processed depending on the priority of the QoS classes.

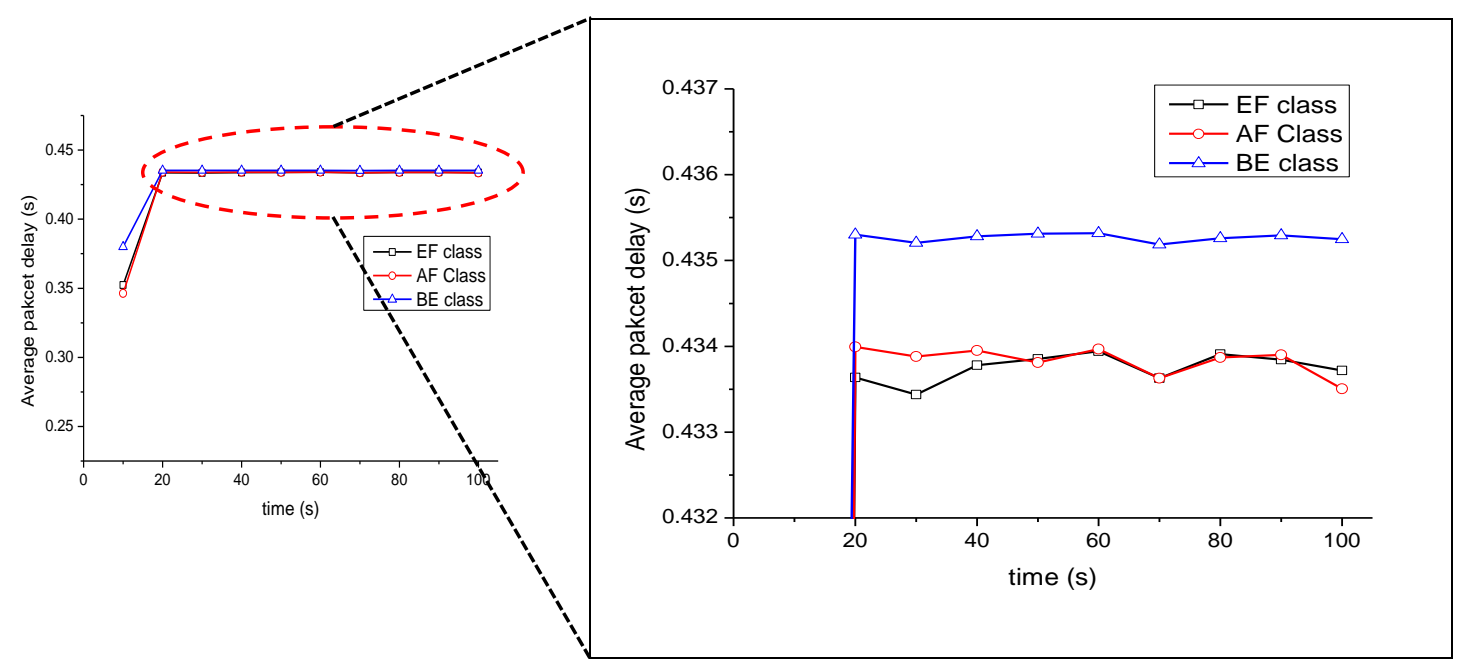

Fig. 10. Round-robin scheduling algorithm

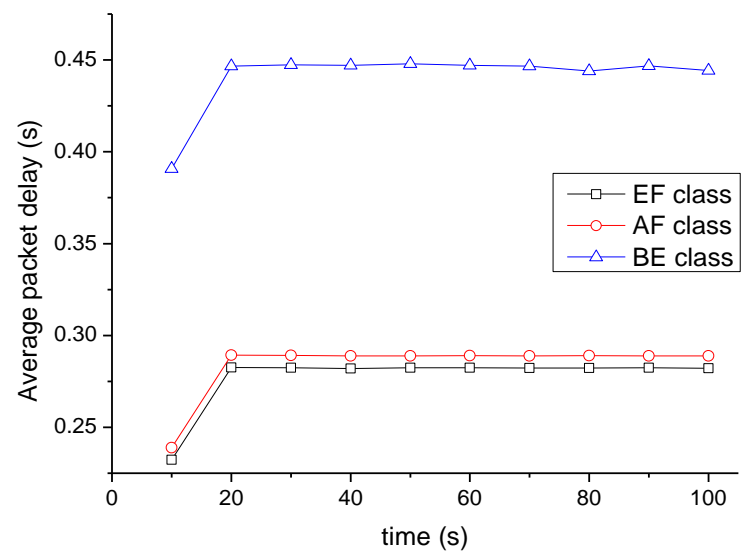

Fig. 11. Priority scheduling algorithm

\subsection{Packet throughput and fairness}

Fairness is one of the most important properties of a computer network. When network resources are unable to satisfy demand, they should be divided fairly between the clients of the 
network. Because the packets are transmitted at different transmission rates in satellite networks using ACM techniques, an unfairness problem is created. To provide fairness to the RCSTs and use the ACM scheme, we share the timeslots with a region with poor weather conditions having low MODCOD.

In this paper, we compared the round-robin and priority scheduling algorithm for the IP layer QoS scheduler to provide a level of QoS differentiation and we also adopt a link layer fairness scheduler to support fairness.

Two cases are considered to demonstrate the sharing of QoS levels and support fairness: $i$ ) throughput per load of traffic, and ii) throughput per time after sufficient traffic has been generated. In other words, $i$ ) case is that resource is enough for each RCST because input traffics are not heavy, but ii) case is that resource is not enough due to heavy input traffics.

\section{A. Round-robin and fairness scheduling}

The round-robin scheduling algorithm is one of the simplest scheduling algorithms for processing packets in network systems. Time slices are assigned to each process in equal portions and in circular order, with all processes handled without priority. The total throughput is equal regardless of the priority of the QoS after the throughput reaches the maximum value. Because the weight value in each MODCOD is perfectly equal, the round-robin scheduling does not offer a guarantee of QoS, as shown in Fig. 12. In addition, although the packets are transmitted to different regions having different MODCODs, which have different transmission rates, we should assure the fairness, as indicated in Fig. 12. As a result of the simulation described in Fig. 13, more timeslots are allocated to regions with poor conditions and low MODCOD than to regions with clear skies and high MODCOD. Therefore, transmitted packets to different regions having different weather conditions are completely equal. Moreover, the amount of transmitted packets to regions having different weather conditions is completely equal regardless of the type of packets.

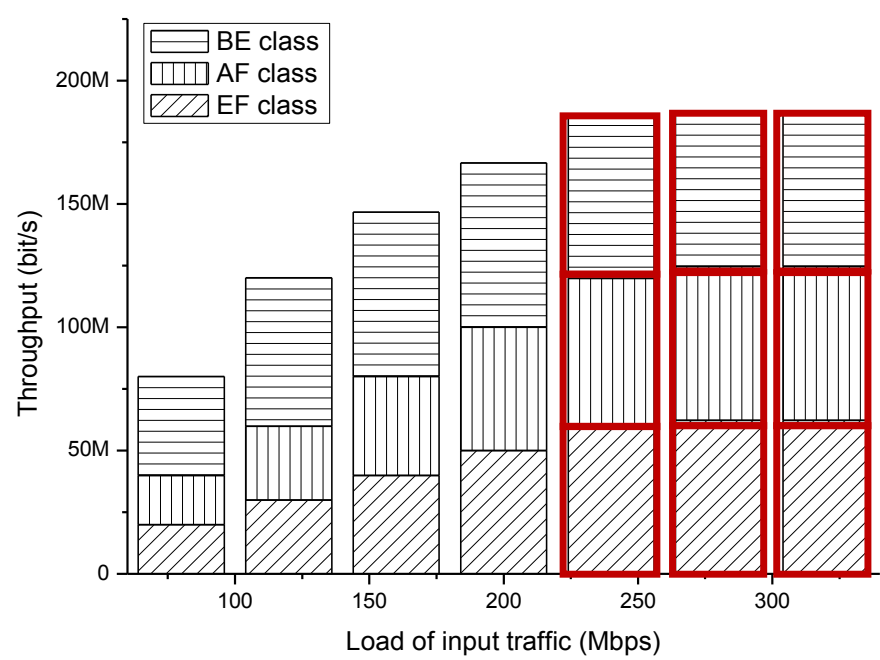

Fig. 12. Throughput per load of input traffic 


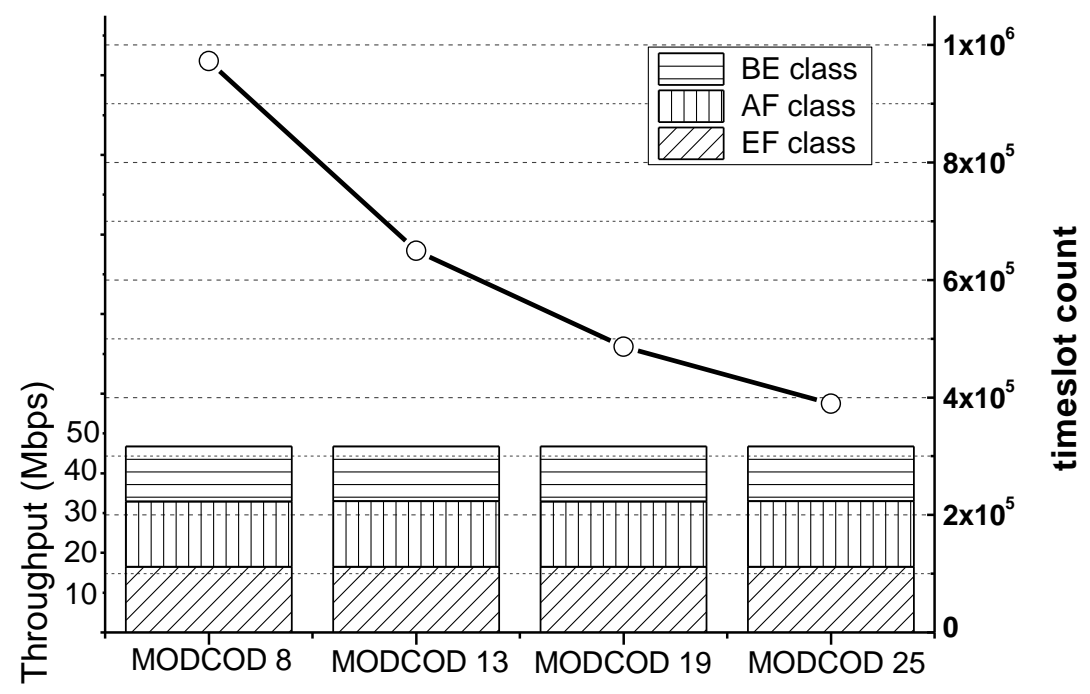

Fig. 13. Throughput of each MODCOD level

\section{B. Priority and fairness scheduling}

The priority scheduling always transmits packets of the highest priority first. If there are no packets of the highest priority level, the next highest priority queue is serviced, and so on. In this paper, the priority scheduling is applied to the IP-layer QoS scheduler to minimize the effect of a decrease in throughput.

Fig. 14 shows the throughput of the priority scheduling. The BE class traffic is gradually decreased when the traffic is increased in the network. Therefore, when the priority scheduling applies to the first-step scheduler, we make up the loss of throughput by supporting fairness for each RCST. The fairness scheduling in the second-step is same with the round-robin scheduling case in terms of fairness. More timeslots are allocated in regions with poor conditions and low MODCOD than in regions with clear skies and high MODCOD, as shown in Fig. 15. Therefore, we offer throughput fairness to each RCST considering different weather conditions. However, the amounts of transmitted packets differ depending on packet types. Although total throughput is decreased due to support throughput fairness for each RCST, we can protect the important data packet using priority scheduling in the first step scheduler. The first scheduler process that high priority packets are always transmitted earlier than low priority packets. As a result of the simulation shown in Fig. 14, more packets of the $\mathrm{EF}$ and AF classes are transmitted than packets of the BE class[23]. 


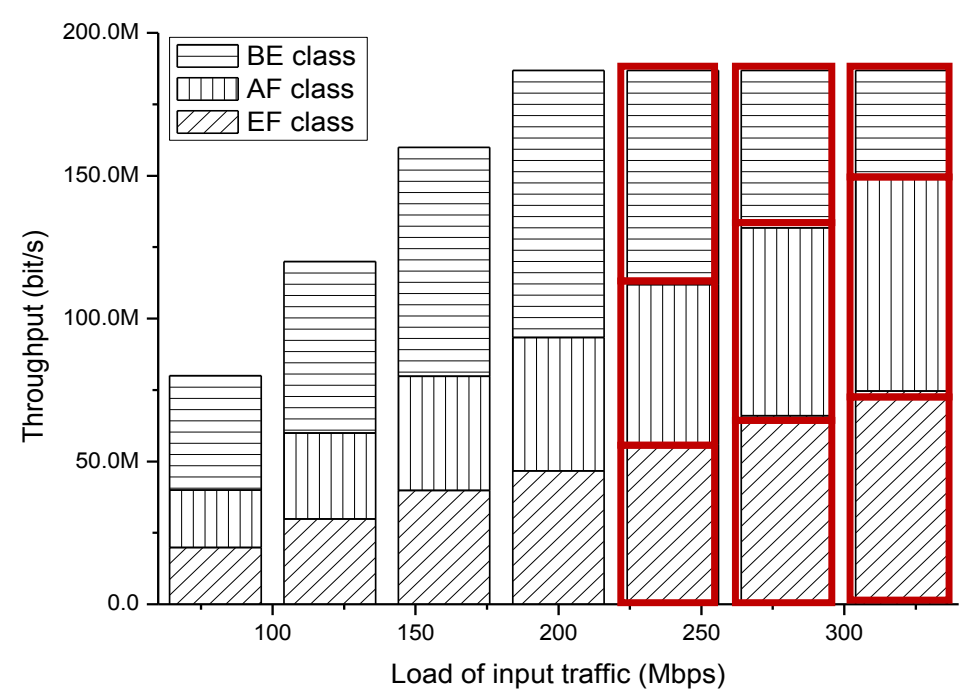

Fig. 14. Throughput per load of input traffic

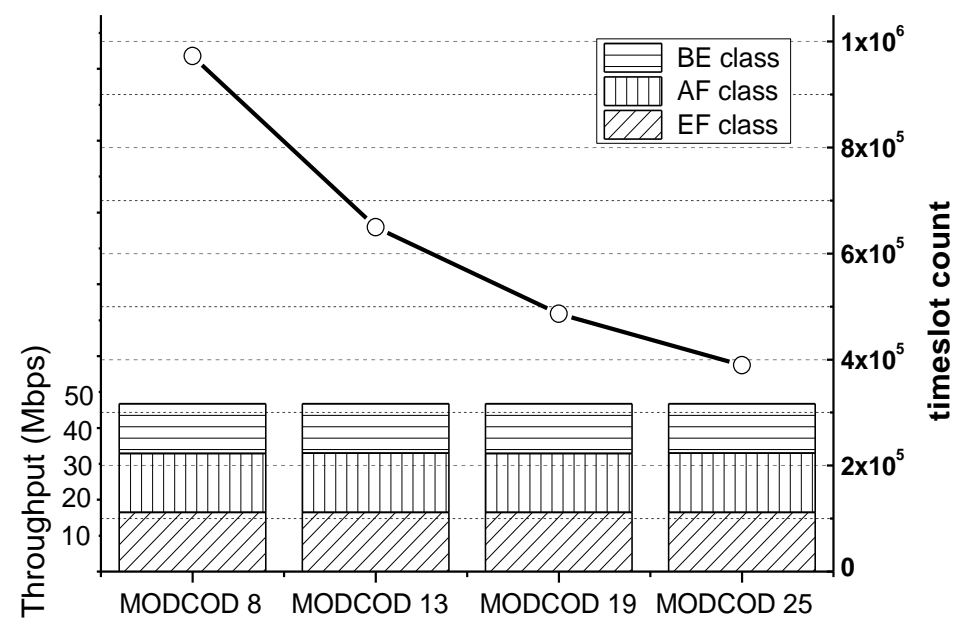

Fig. 15. Throughput of each MODCOD level

\section{Conclusion}

DVB-S2 satellite communication system including ACM leads to greater spectrum efficiency since the transmission parameters are dynamically adjusted to accommodate the channel conditions. Absolutely, satellite communication system with ACM scheme has higher transmission efficiency, but it does not offer earth station fairness because the data are transmitted with high MODCOD in regions with clear skies, and are transmitted with low MODCOD in regions experiencing rain event. 
In this paper, we propose a two-step scheduling scheme to supporting fairness and QoS in DVB-S2 ACM systems. To achieve our goals which throughput fairness and Qos support, we propose fairness improvement-weighted round robin (FI-WRR) scheduler which the allocation timeslot is calculated by the transmission rate of each MODCOD queue. The proposed scheduler can be used to offer earth station fairness but it allows to be decreased the total system performance.

DVB-S2 ACM system has to be adopted QoS mechanism because of bandwidth fluctuation according to the various MODCODs. To overcome this problem, we adopt priority scheduling for QoS support at the IP layer, it carry out packet processing before operating the link layer fairness scheduler. The simulation results show that the proposed scheme using the priority and fairness scheduling can be used to offer earth stations fairness and QoS. And the priority scheduler at the IP layer can be useful to protect the important packets for a decreased throughput owing to its fairness support.

\section{References}

[1] ETSI EN 302307 v1.1.1, Digital Video Broadcasting (DVB); Second Generation Framing Structure, Channel Coding and Modulation Systems for Broadcasting, Interactive Services, News Gathering. ETSI, June 2004. Article (CrossRef Link)

[2] ETSI TR 101790 V1.4.1, Digital Video Broadcasting (DVB): Interaction channel for satellite distribution systems. Guidelines for the use of EN 301 790, July 2009. Article (CrossRef Link)

[3] A. Morello and V. Mignone, "DVB-S2: the second generation standard for satellite broad-band services," in Proc. of the IEEE, vol. 94, no. 1, Jan. 2006. Article (CrossRef Link)

[4] H. Bischl et al., "Adaptive coding and modulation for satellite broadband networks: From theory to practice,” Int. J. Satellite Communications and Networking, vol. 28, no. 2, pp. 59-111, 2010. Article (CrossRef Link)

[5] ETSI EN 300421 v1.1.2, Digital Video Broadcasting (DVB); Framing Structure, Channel Coding and Modulation for 11/12 GHz Satellite Services. ETSI, Aug. 1997. Article (CrossRef Link)

[6] M.A.V.C Vázquez Castro, F. V. Vieira, "DVB-S2 full cross-layer design for QoS provision," IEEE Communications Magazine, Vol. 50 , No. 1 , pp. 128-135, Jan. 2012. Article (CrossRef Link)

[7] F. Vieira, M. A. Vazques Castro, and G. Seco Granados, "A tunable-fairness cross-layer scheduler for DVB-S2,” Int. J.Satellite Communications and Networking, vol. 24, pp.437-450, 2006. Article (CrossRef Link)

[8] S. Blake et al., RFC 2475: An Architecture for Differentiated Service, 1998. Article (CrossRef Link)

[9] E. Rendon-Morales, "Adaptive Packet Scheduling for the support of QoS over DVB-S2 Satellite Systems," 9th International Conference on Wired/Wireless Internet Communications (WWIC 2011), vol. 6649, pp.15-26, 2011. Article (CrossRef Link)

[10] ManKyu Park, DeockGil Oh, "Cross-layer design for improving TCP PEP performance in DVB-RCS2 networks," ICT Convergence (ICTC) 2013 proceeding, pp. 864-847, 2013. Article (CrossRef Link)

[11] Lei Xu, Dazhuan Xu, Xiaofei Zhang, and Shufang Xu, "Cross-Layer Resource Allocation Scheme for WLANs with Multipacket Reception,” ETRI Journal, vol.33, no.2, pp.184-193, Apr. 2011. Article (CrossRef Link)

[12] ETSI TS 102 462, ETSI Standard for Satellite Earth Stations and Systems (SES); Broadband Satellite Multimedia (BSM); QoS Functional Architecture 2006. Article (CrossRef Link)

[13] ETSI TS 102 292: "Satellite Earth Stations and Systems (SES); Broadband Satellite Multimedia (BSM) services and architectures; Functional architecture for IP interworking with BSM networks". Article (CrossRef Link)

[14] Emanuel B. Rodrigues and Fernando Casadevall, Control of the Trade-Off between Resource Efficiency and User Fairness in Wireless Networks Using Utility-Based Adaptive Resource 
Allocation, IEEE Communications Magazine September 201, pp. 90-98. Article (CrossRef Link)

[15] J.-Y. Le Boundec and B. Radunovic, "Rate performance objectives of multihop wireless networks," IEEE transactions on Mobile computing, 3, pp. 334-349, 2004. Article (CrossRef Link)

[16] Unhee Park, Hee Wook Kim, Dae Sub Oh, and Bon-Jun Ku, "Interference-Limited Dynamic Resource Management for an Integrated Satellite/Terrestrial System,” ETRI Journal, Vol. 36, no. 4, Aug. 2014, pp. 519-527. Article (CrossRef Link)

[17] Emanuel B. Rodrigues, Fernando Casadevall, Pawel Sroka, Marco Moretti and Giulio Dainelli, "Resource Allocation and Packet Scheduling in OFDMA-Based Cellular Networks," in Proc. of The 4th International Conference on CROWNCOM, 2009. Article (CrossRef Link)

[18] M. Shreedhar and G. Varghese, "Efficient fair queuing using deficit round robin," IEEE/ACM Transaction on Networking, vol. 4, June 1996, pp.375-385. Article (CrossRef Link)

[19] L. Kleinrock. Queuing Systems, Volume 1. Wiley, NewYork, 1975. Article (CrossRef Link)

[20] J. Schmitt, "On Average and Worst Case Behavior in Non-Preemptive Priority Queuing," International Symposium on Performance Evaluation of Computer and Telecommunication Systems, 2003. Article (CrossRef Link)

[21] Robert M. Cubert and Dr. Paul Fishwick, sim++ Version 1.0 manual, 28 July, 1995. Article (CrossRef Link)

[22] IETF RFC 3140, Per Hop Behavior Identification Codes, June 2001. Article (CrossRef Link)

[23] ManKyu Park, et al., "Hierarchical Packet Scheduler for Supporting Fairness and QoS Over DVB-S2 ACM Systems," ICCE, 2013. Article (CrossRef Link)

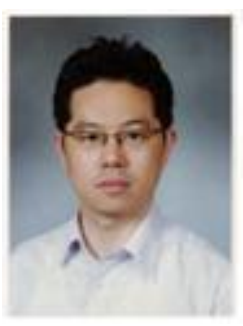

ManKyu Park is a senior member of engineering staff in aerospace ICT research department, Electronics and Telecommunications Research Institute (ETRI). He received the B.S. and M.S. degrees, from Kongju National University, Korea in 1999 and 2001, respectively. He received the $\mathrm{Ph} . \mathrm{D}$. degree in computer networks from Chungnam National University, Korea in 2011. He joined ETRI in 2009, his research interests include Internet protocols, traffic control, TCP congestion control, and system design/development for satellite communication and broadcasting systems.

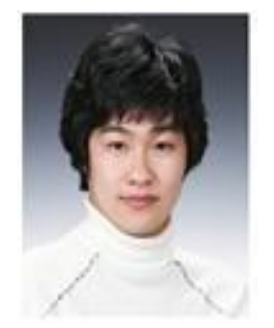

DongBae Kang is a member of engineering staff in Agency for Defense Development (ADD). He received the B.S. degree in computer engineering from Chosun University, Korean in 2011, and M.S. degree in mobile communication \& digital broadcasting engineering from University of Science \& Technology (UST), Korea, in 2013. His research interests include Internet protocols, and satellite \& mobile communication systems.

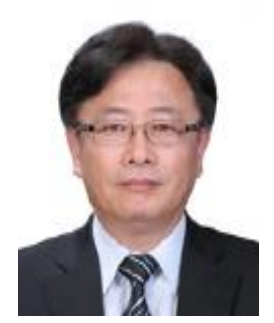

DeockGil Oh is a team leader at satellite broadcasting and communication convergence team. He received the B.S., M.S., and Ph.D. degree in Electronics Engineering from Seoul National University, Korea in 1980, 1984, and 1996, respectively. He joined Electronics and Telecommunications Research Institute (ETRI) in 1982, he mainly carries on the research of next generation satellite communication and broadcasting systems such as DVB-RCS2, DVB-S2, DVB-S2X systems. 